\title{
ANALYSIS OF INFLUENCING FACTORS ON WINTER WHEAT YIELD ESTIMATIONS BASED ON A Multisource REMOTE SENSING DATA Fusion
}

\author{
Yan Li ${ }^{1,2}$, Yan Z. Ren ${ }^{3}$, Wan L. Gao ${ }^{1,4}$, Sha Tao ${ }^{1,4, *}$ Jing D. Jia ${ }^{4}$, Xin L. Liu ${ }^{1,5, * *}$ \\ College of Information and Electrical Engineering, China Agricultural University, Beijing, China. \\ College of Urban Construction, Hebei Normal University of Science and Technology, Qinhuangdao, Hebei, China. \\ College of Science, China Agricultural University, Beijing, China. \\ Key Laboratory of Agricultural Information Standardization, Ministry of Agriculture and Rural Affairs, Beijing, China. \\ 5 National Engineering Laboratory for Agri-product Quality Traceability, Beijing Technology and Business University, Beijing, China. \\ ,*** Correspondence: *taosha20070608@163.com, ${ }^{* *}$ liuxinl@btbu.edu.cn.
}

\section{Highlights}

- The potential of fusing GF-1 WFV and MODIS data by the ESTARFM algorithm was demonstrated.

- A better time window selection method for estimating yields was provided.

- A better vegetation index suitable for yield estimation based on spatiotemporally fused data was identified.

- The effect of the spatial resolution of remote sensing data on yield estimations was visualized.

\begin{abstract}
The accurate estimation of crop yields is very important for crop management and food security. Although many methods have been developed based on single remote sensing data sources, advances are still needed to exploit multisource remote sensing data with higher spatial and temporal resolution. More suitable time window selection methods and vegetation indexes, both of which are critical for yield estimations, have not been fully considered. In this article, the Chinese GaoFen-1 Wide Field View (GF-1 WFV) and Terra Moderate Resolution Imaging Spectroradiometer (MODIS) data were fused by the Enhanced Spatial and Temporal Adaptive Reflectance Fusion Model (ESTARFM) to generate time-series data with a high spatial resolution. Then, two time window selection methods involving distinguishing or not distinguishing the growth stages during the monitoring period, and three vegetation indexes, the normalized difference vegetation index (NDVI), two-band enhanced vegetation index (EVI2) and wide dynamic range vegetation index (WDRVI), were intercompared. Furthermore, the yield estimations obtained from two different spatial resolutions of fused data and MODIS data were analyzed. The results indicate that taking the growth stage as the time window unit division basis can allow a better estimation of winter wheat yield; and that WDRVI is more suitable for yield estimations than NDVI or EVI2. This study demonstrates that the spatial resolution has a great influence on yield estimations; further, this study identifies a better time window selection method and vegetation index for improving the accuracy of yield estimations based on a multisource remote sensing data fusion.
\end{abstract}

Keywords. Remote sensing, Spatiotemporal data fusion, Winter wheat, Yield estimation.

W heat is among the crops with the widest cultivation areas and the highest commodity rates in the world, and timely and reliable winter wheat yield estimations are highly required, considering the great significance of these estimations in guiding agricultural production and ensuring food security (He et al., 2010; Curtis and Halford, 2014; Lopez et al., 2020).

Moderate Resolution Imaging Spectroradiometer (MODIS) is a key instrument aboard the Terra and Aqua satellites. Terra MODIS and Aqua MODIS view the entire

Submitted for review on 16 November 2020 as manuscript number ITSC 14398; approved for publication as a Research Article and as part of the Computer Modeling and Statistics for Agriculture Collection by the Information Technology, Sensors, \& Control Systems Community of ASABE on 23 April 2021.
Earth's surface every 1 to 2 days, acquiring data in 36 spectral bands or groups of wavelengths. These data improve our understanding of global dynamics and processes occurring on land, in the oceans, and in the lower atmosphere. At present, MODIS data have been widely used in crop growth monitoring and yield estimations at the regional scale (Chen et al., 2016; Huang et al., 2016; Karthikeyan et al., 2020). However, the mixed pixel phenomenon is critical in monitoring areas with scattered and mixed crops due to the relatively low spatial resolution of MODIS $(250-1000 \mathrm{~m})$, affecting the accuracy of yield estimation. Therefore, data fusion methods have been introduced in many studies. For example, Gao $(2006,2018)$ proposed a spatial and temporal adaptive reflectance fusion model (STARFM) and verified that the use of a combination of Landsat and MODIS data better explain the spatial variability of a crop yield than the use of a single data source alone. 
In terms of data sources, most research has focused on the integration of Landsat and MODIS data (Emelyanova et al., 2013; Chen et al., 2020; Xu et al., 2020; Zhao et al., 2020). The GaoFen-1 satellite is equipped with four 16-m resolution multispectral wide-format cameras (GF-1 WFV) with a temporal resolution of 4 days. Compared with Landsat, the GF-1 WFV satellite has more opportunities to obtain cloudless data. At present, relevant studies have begun to fuse GF1 and MODIS data to obtain serial remote sensing data with high spatial and temporal resolutions (Kong et al., 2016; Tao et al., 2019). However, the normalized difference vegetation index (NDVI) and enhanced vegetation index (EVI) are still the most commonly used indexes in vegetation index selections. Although some scholars have compared the impacts of NDVI, EVI, the normalized difference water index (NDWI), the land surface water index (LSWI) and other vegetation indexes on yield estimations (Sakamoto, 2020; Beniaich et al., 2019; Wang et al., 2014), these studies are still based on MODIS data sources, and there are few comparative studies on vegetation indexes obtained from remote sensing data fusions with higher spatial resolutions (Gao and Anderson, 2019; Zhang et al., 2016). Gao and Anderson (2019) demonstrated that EVI2 better explains the variability in crop yields than NDVI through only the comparison of NDVI and EVI2 using Landsat-Sentinel2-MODIS fused data, and Zhang et al. (2016) assimilated more vegetation indexes into a wheat growth model using a combination of resampled SPOT and Landsat data. However, the wide dynamic range vegetation index (WDRVI), which introduces a weighting coefficient to the NIR band reflectance and has a wider dynamic range than NDVI in densely vegetated areas, was ignored. Furthermore, meteorological factors have not been integrated in previous studies. Based on this, GF-1 WFV and Terra MODIS data were fused in this study to construct time-series remote sensing data with a spatial resolution of $16 \mathrm{~m}$. Time-series meteorological information was integrated to estimate winter wheat yield. The purpose of this study is to compare the differences between the use of fused data and the use of MODIS data in the accuracies of yield estimations and to determine which vegetation index from the fine-resolution fused data, other than the commonly used NDVI, can provide the best accuracy for yield estimations.

In addition to the data fusion and vegetation index selection, determining the optimal time window for crop yield estimations is a key issue in achieving accurate estimation results. The morphological and physiological characteristics of crops vary at different growth stages. Previous studies have shown that there are significant differences in the effects of the growth status and environmental conditions that occur at different growth stages on yield formations (Khanizadeh et al., 2005; Zou and Mottus, 2017). By dividing the growth cycle of maize into four key growth stages, Wang (2020) explored the impacts of corn growth and drought on yield at each growth stage. The results showed that the heading-milk stage and jointing-heading stage have the greatest impacts, followed by the milk-mature stage, and the seedling-joining stage has the smallest impact.

However, in previous studies, the optimal monitoring time windows for yield estimations were still selected according to month (Han et al., 2020; Lin and Shao, 2020), and the effect of using growth stages as the time windows was not considered. Therefore, in terms of the time window selection, the growth stage was taken as the basis for the time unit division in this study, and based on discussing the data source and vegetation index, the influence of dividing the time units according to the growth stage on the yield estimation accuracy was further explored.

\section{Materials ANd Methods \\ STUDY AREA}

The study area extends from $114^{\circ} 01^{\prime} \mathrm{E}$ to $117^{\circ} 57^{\prime} \mathrm{E}$ and from $37^{\circ} 03^{\prime} \mathrm{N}$ to $38^{\circ} 57^{\prime} \mathrm{N}$, consisting of 45 counties (districts) in Cangzhou City, Hengshui City, and Shijiazhuang City covering the main winter wheat planting area in the central plain of Hebei Province in China (fig. 1). This region has a subhumid climate with an average temperature ranging from $4^{\circ} \mathrm{C}$ to $13^{\circ} \mathrm{C}$ and annual average precipitation between 400 and $900 \mathrm{~mm}$. The area is high in the northwest and low in the southeast. The elevation is between 0 and $100 \mathrm{~m}$. The soil types are mainly fluvo-aquic soils, wet fluvo-aquic soils and sandy fluvo-aquic soils. The cropping pattern of the area consists of winter wheat in rotation with summer maize (Jiang et al., 2014). For winter wheat, the growing cycle occurs from early October of a given year to mid-June of the following year and is divided into the green-up stage, the jointing stage, the heading-filling stage and the milk stage. Specifically, the green-up stage begins from approximately early March to mid-March, the jointing stage is from approximately late March to mid-April, the heading-filling stage lasts from approximately late April to early May, and the milk stage occurs from approximately mid-May to late May (Pan et al., 2019).

\section{Datasets and Processing \\ Remote Sensing Data}

The GF-1 WFV and Terra MODIS data collected in 2017 were obtained for the winter wheat planting area extraction, spatiotemporal data fusion, vegetation index calculation and winter wheat yield estimation conducted in this study. The growth period of winter wheat, from the green-up stage to the milk stage, lasts from early March to late May; thus, only the data collected during this period were utilized for the yield estimations. The GF-1 WFV data were downloaded from the China Center for Resources Satellite Data and Application (http://36.112.130.153:7777/DSSPlatform/index.html), and the Terra MODIS data were obtained from the National Aeronautics and Space Administration (NASA) (https://ladsweb.modaps.eosdis.nasa.gov/search/). Among them, MOD09GQ is a daily surface reflectance product with a spatial resolution of $250 \mathrm{~m}$, and MOD09Q1 is a surface reflectance product synthesized in 8 days with a spatial resolution of $250 \mathrm{~m}$. The acquisition dates of the GF-1 WFV and MOD09GQ data covering the entire study area, which were acquired in the morning under clear-sky conditions, are shown in table 1. Data were collected from 4 days of overpasses by both GF-1 WFV and MOD09GQ, and the data acquired during these 4 days were utilized in the spatiotemporal data fusion and in the fusion accuracy 


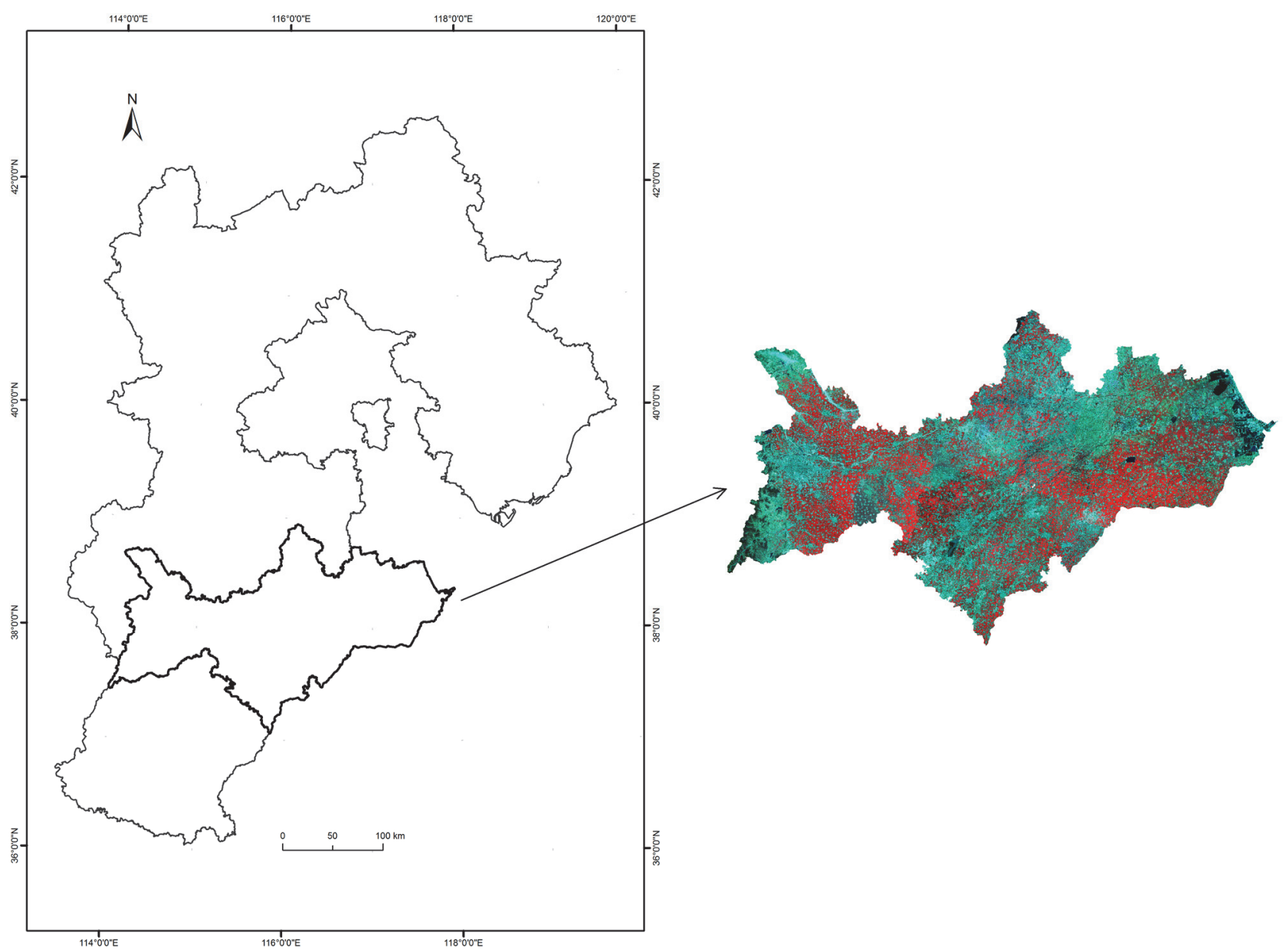

Figure 1. The location of the study area in Hebei Province, China. The right figure is a false-color composite image of the study area on 18 April 2017 using bands 2, 3, and 4 of GF-1 WFV data.

verification. To reduce the effects of the solar elevation angle, satellite observation angle, orbit drift, clouds and shadows, the MOD09Q1 product covering the period from March to May was obtained as the coarse-resolution data for the spatiotemporal data fusion over the period to be predicted. In addition, the MOD09Q1 product, with an 8-day interval, was used as the coarse-resolution data source for the yield estimations to compare the yield estimation results with those obtained based on the fused fine-spatial-resolution data.

Table 1. Acquisition dates of the GF-1 WFV and MOD09GQ data under clear-sky conditions.

\begin{tabular}{cccccc}
\hline \multirow{2}{*}{ City } & Data Source & Green-up $^{[\mathrm{a}]}$ & Jointing $^{[\mathrm{b}]}$ & Heading- & \\
Filling & & Milk $^{[\mathrm{d}]}$ \\
\hline Shijiazhuang & GF-1 WFV & March 7, & April 1, & April 26 & May 12 \\
& & March 12 & April 18 & & \\
& MOD09GQ & March 7 & April 18 & April 26 & May 12 \\
\hline Hengshui & GF-1 WFV & March 8 & April 18 & April 26 & May 12, \\
& & & & & May 16 \\
& MOD09GQ & March 8 & April 18 & April 26 & May 12 \\
\hline Cangzhou & GF-1 WFV & March 7, & April 18 & April 26 & May 12, \\
& & March 12 & & & May 16 \\
& MOD09GQ & March 7 & April 18 & April 26 & May 16 \\
\hline [a]
\end{tabular}

\footnotetext{
[a] Green-up stage.

[b] Jointing stage.

[c] Heading-filling stage.

[d] Milk stage.
}

The acquired GF-1 WFV images were preprocessed by radiometric calibration, atmospheric correction and geometric correction, stitching, and cropping, and were then used to calculate the vegetation indexes. The MOD09 products are standard level-2 products that have been geometrically and atmospherically corrected. The MODIS data were reprojected and resampled to the projection (UTM zone $49 \mathrm{~N}$ ) and resolution $(16 \mathrm{~m})$ of the GF-1 WFV data for the spatiotemporal data fusion. The red and near-infrared (NIR) bands of the GF-1 WFV data and the corresponding bands in the MODIS data are shown in table 2.

NDVI, EVI2, and WDRVI have good correlations with crop yield, so they are often used in crop yield estimations. However, when the vegetation coverage is high and the leaf area index (LAI) is large (LAI $>3$ ), NDVI is easily saturated (Du et al., 2013), while EVI2 and WDRVI can reduce the canopy background signal and atmospheric influence and enhance the dynamic monitoring effect of vegetation in high-

Table 2. GF-1 WFV bands and the corresponding Terra MODIS bands used for data fusion.

\begin{tabular}{lcc}
\hline Data Source & Band & Bandwidth $(\mathrm{nm})$ \\
\hline GF-1 WFV & 1(Red) & $630 \sim 690$ \\
& 2(NIR) & $770 \sim 890$ \\
\hline MOD09GQ/MOD09Q1 & 1(Red) & $620 \sim 670$ \\
& 2(NIR) & $841 \sim 876$ \\
\hline
\end{tabular}


biomass areas. According to equations 1-3, NDVI, EVI2, and WDRVI were calculated with the red and NIR band data to compare their estimation abilities for winter wheat yield.

$$
\begin{gathered}
\mathrm{NDVI}=\frac{\rho_{N I R}-\rho_{\text {Red }}}{\rho_{N I R}+\rho_{\text {Red }}} \\
\mathrm{EVI} 2=\frac{2.5\left(\rho_{N I R}-\rho_{R e d}\right)}{\rho_{N I R}+2.4^{*} \rho_{R e d+1}} \\
\mathrm{WDRVI}=\frac{0.1 \rho_{N I R}-\rho_{\text {Red }}}{0.1 \rho_{N I R}+\rho_{\text {Red }}}
\end{gathered}
$$

where

$\rho_{N I R}=$ the surface reflectance of the NIR band,

$\rho_{\text {Red }}=$ the surface reflectance of the red band.

\section{Meteorological Data}

Meteorological conditions have important impacts on crop growth and yield. In the context of global climate change, extreme temperatures and droughts have adverse effects on crops. Referring to previous studies (Kern et al., 2018; Wheeler et al., 2000), the average temperature ( $\left.T_{\text {mean }}\right)$, maximum temperature $\left(T_{\max }\right)$, minimum temperature $\left(T_{\min }\right)$, precipitation (PRE) and surface temperature (GST) were selected as meteorological factors to participate in the crop yield estimations. Meteorological data were obtained from the China Meteorological Science Data Sharing Service Network (http://data.cma.cn/). Daily meteorological observation data spanning the period from March to May 2017 were extracted. The average values of each selected factor at the green-up stage, jointing stage, heading-filling stage and milk stage were calculated, and meteorological data with a $16-\mathrm{m}$ spatial resolution for each county (district) were generated through the interpolation method of the geographic information system.

\section{Winter Wheat Yield Data and Planting Area}

The unit-area winter wheat yield data were recorded in the 2018 statistical yearbooks of each city. The winter wheat planting areas were extracted using the decision tree classification method according to the change law of winter wheat NDVI during the phenological period (Zhang et al., 2019; Zhou et al., 2020). Winter wheat was the only summer harvest in the study area. From early March to mid-March, winter wheat entered the green-up stage and grew rapidly, with $\mathrm{NDVI}>0.3$. By the end of April, the growth of winter wheat was the most vigorous, and it was harvested in succession from early June to mid-June, so the NDVI in late April was greater than that in early June. The winter wheat planting areas are shown in figure 2. Compared with the statistical area reported in the 2018 statistical yearbooks, the classification accuracies in 17 regions (mainly located in Shijiazhuang City and Cangzhou City) were between $80 \%$ and $90 \%$, and the accuracies were higher than $90 \%$ in other regions. These classification accuracies can meet the demand for yield estimations.

\section{MeTHODS}

The framework of the methods followed in this study includes four components: the spatiotemporal data fusion, the

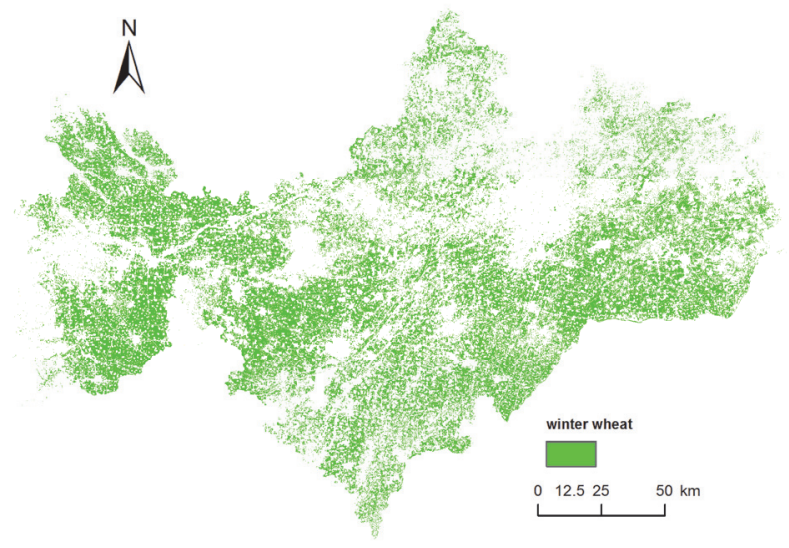

Figure 2. Distribution of winter wheat in the study area in 2017.

acquisition of variables for the yield estimation models, the comparison and identification of the factors affecting the yield estimations, and the analysis of the yield estimation results obtained from different spatial resolutions. The GF-1 WFV and Terra MODIS data were fused using the ESTARFM algorithm to generate time-series images with high spatial and temporal resolutions covering the main growth periods of winter wheat. NDVI, EVI2, and WDRVI were calculated based on the fused time-series data and MOD09Q1 time-series data, respectively, and smoothed using the Savitzky-Golay filter method. The meteorological factors were obtained using the spatial interpolation method. A better time window selection method and a better vegetation index for winter wheat yield estimations were identified based on the random forest regression (RFR) method. The yield estimation results obtained using remote sensing data with different spatial resolutions were compared and analyzed.

\section{Spatiotemporal Data Fusion}

Zhu et al. (2010) described the principle and usage of the ESTARFM data fusion algorithm in detail. ESTARFM requires two pairs of fine-spatial-resolution images and coarsespatial-resolution images acquired on the same dates and a series of coarse-spatial-resolution images obtained on the dates to be predicted. According to the acquisition dates of the GF-1 WFV and Terra MODIS data (table 1), the implementation of the ESTARFM algorithm was divided into two segments in this study. From the green-up stage to the jointing stage, the GF-1 WFV and Terra MODIS data acquired on 7 March (8 March in Hengshui City) and 18 April were used as the input image pairs in ESTARFM, and the Terra MODIS data representing each 8-day interval were selected as the coarse-spatial-resolution data for the period to be predicted. From the heading-filling stage to the milk stage, the GF-1 WFV and Terra MODIS data acquired on 18 April and 12 May were used as the input image pairs in ESTARFM, and the Terra MODIS data representing each 8-day interval were selected as the coarse-spatial-resolution data for the period to be predicted. When the cloudiness of the GF-1 WFV images exceeded $10 \%$, the spatiotemporally fused data were adopted. The dates occurring after early May were far from the date when high-quality images were obtained, so their prediction uncertainties were large. The images taken in late 


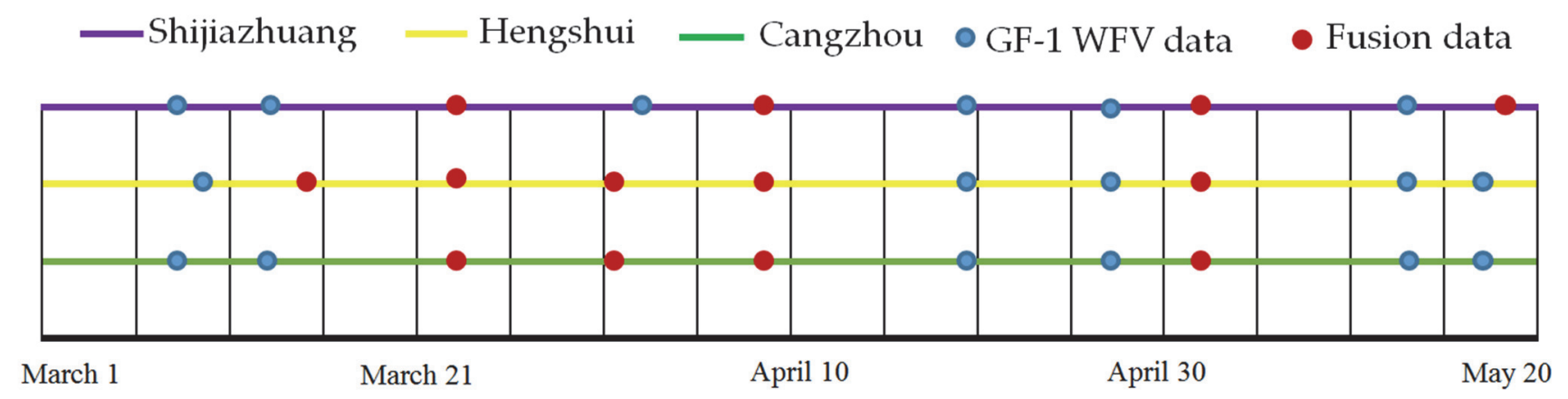

Figure 3. Date distribution of the remote sensing image sequence during the main growth period of winter wheat.

May were not fused in this article. The temporal distribution of the final remote sensing image sequence is shown in figure 3. The GF-1 WFV image obtained on 26 April and the MOD09Q1 products obtained on 22 March, 7 April, and 26 April were selected to test the prediction accuracy of ESTARFM.

ESTARFM is a data fusion method that is based on a moving window that considers the auxiliary information obtained from adjacent pixels with similar spectra and the weights of these pixels themselves. First, two-phase GF-1 WFV images were used to search for pixels similar to the central pixel in a given local window. Then, the weight $\left(W_{i}\right)$ of each similar pixel and the conversion coefficient $\left(V_{i}\right)$ were assigned according to the two-phase MOD09GQ data. Finally, $W_{i}$ and $V_{i}$ were used to calculate the fine-resolution reflectance from the MOD09Q1 data at the desired prediction date. The input parameters in ESTARFM mainly include the number of classes and the size of the moving window. According to the homogeneity of the land surface in the study area and according to related research (Zhou et al., 2020; Zhu et al., 2010), the number of classes was set to 10 , and the window size radius was set to 25 .

\section{RFR Method for Estimating Winter Wheat Yield}

The RFR method uses an ensemble of decision trees, which usually vote or are averaged to obtain the final result (Zouggar and Adla, 2019; Rastgou et al., 2020). RFR does not easily fall into overfitting and is more robust than other methods in terms of noise due to the introduction of randomness. Many studies have shown that the RFR method has good applicability in crop yield estimations (Breiman, 2001; Alberto et al., 2014; Franz et al., 2020).

The process of the RFR method to estimate winter wheat yield is as follows.

1. During the monitoring period, NDVI, EVI2, and WDRVI were combined with meteorological factors as input variables in the model, and the winter wheat yield at the country scale was the target vector of the model, totaling 45 samples. $n$ training sample subsets were obtained, and $\mathrm{n}$ regression trees were generated through bootstrap resampling. In each bootstrap resampling, the unselected data were close to $1 / \mathrm{e}$, approximately 0.37 ; that is, approximately $37 \%$ of the training data were not used in the construction of a single model. These data are called outof-bag (OOB) data. The generalization ability of the model was tested based on the OOB prediction error, and the test set was not needed. The $\mathrm{n}$ training samples obtained by the bagging method were not the same, which ensured the difference of the regression trees.

2 . In the growth process of each regression tree, when each node was split, $M$ variables (RFR generally selects $1 / 3$ of the total variables, so $M$ was 1) were randomly selected for splitting based on the classification and regression tree (CART) method, and no pruning was required during the splitting process.

3. Each regression tree split and grew from top to bottom, and the estimated value was set as the output until the tree reached a certain leaf node. All regression trees were amalgamated to a random forest at the end of the process. All winter wheat yield estimates output by the regression trees were averaged to serve as the final estimation results.

\section{Identifying a Better Time Window Selection Method for Winter Wheat Yield Estimations}

There are usually two ways to select a monitoring time window for crop yield estimations: one is to select a certain period by month during the crop growth cycle without distinguishing the growth stage, and in this method, the observed variables in the whole monitoring period are averaged; the other is to divide the crop growth cycle into several key growth stages, and in this method, the observed variables in each growth stage are averaged. The former method treats the selected monitoring period as a single time window, while the latter divides the monitoring period into multiple time windows according to the growth stages. The growth status and environmental conditions of each growth stage have different effects on yield formation. Taking the growth stage as the time window unit division basis instead of taking the entire growth process as a single time window may allow a better estimation of winter wheat yield. To compare the resulting differences between the use of a single time window and multiple time windows for winter wheat yield estimations and to analyze the impacts of remote sensing and meteorological observation variables in the late growth stage (heading-filling stage and milk stage) on the accuracies of yield estimations, the growth cycle of winter wheat after overwintering (from March to May) was attributed to four monitoring time strategies, and corresponding winter wheat yield estimation models were established 
as follows: (1) a model based on four time windows, the green-up stage, jointing stage, heading-filling stage and milk stage; (2) a model based on only three time windows, the green-up stage, jointing stage and heading-filling stage; (3) a model based on a single time window in which the variables from four growth stages - the green-up stage, jointing stage, heading-filling stage, and milk stage - were averaged; and (4) a model based on a single time window in which the variables from three growth stages - the green-up stage, jointing stage, and heading-filling stage-were averaged. The accuracies of the winter wheat yield estimation models designed using the four time strategies were compared, and then an appropriate time strategy was identified.

\section{Identifying Better Vegetation Indexes for Winter Wheat Yield Estimations}

Although many vegetation indexes have been developed for monitoring vegetation changes (Gitelson, 2004; Jiang et al., 2008), NDVI and EVI are still the two most commonly used vegetation indexes for crop yield estimations because of their relatively easy acquisition. WDRVI, which has shown a strong linear relationship with corn green LAI (Sakamoto et al., 2013) may be able to estimate winter wheat yield better than NDVI or EVI. To compare the yield estimation capabilities of NDVI, EVI2, and WDRVI during the main growth period of winter wheat and the impacts of meteorological factors on the yield estimation results, six combination schemes were selected as input variables of RFR, and winter wheat yield estimation models were established based on the determined optimal time strategy. First, regardless of the meteorological factors, the NDVI model for estimating winter wheat yield was established by considering only NDVI at each growth stage; the EVI2 model for estimating winter wheat yield was established by considering only EVI2 at each growth stage; and the WDRVI model was established by considering only WDRVI at each growth stage. Then, by integrating the meteorological factors and each vegetation index, yield estimation models were established-namely, the NDVI+ meteorology model, EVI2+ meteorology model and WDRVI+ meteorology model.

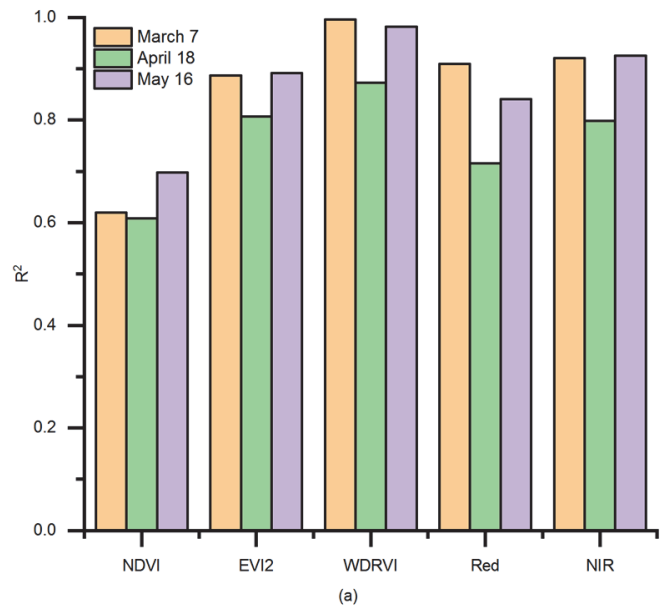

\section{Model Evaluation}

The models established for different input variables were cross-validated by the tenfold crossover method. The accuracies of the model predictions were evaluated using the coefficient of determination $\left(\mathrm{R}^{2}\right)$, root mean square error (RMSE), and mean absolute error (MAE).

$$
\begin{gathered}
\mathrm{R}^{2}=\left[\frac{\sum_{i=1}^{n}\left(y_{i}-\overline{y_{i}}\right)\left(y_{i}^{\prime}-\overline{y_{i}^{\prime}}\right)}{\sum_{i=1}^{n}\left(y_{i}-\overline{y_{i}}\right) \sum_{i=1}^{n}\left(y_{i}^{\prime}-\overline{y_{i}^{\prime}}\right)}\right]^{2} \\
\mathrm{RMSE}=\sqrt{\frac{1}{n} \sum_{i=1}^{n}\left(y_{i}-y_{i}^{\prime}\right)^{2}} \\
\operatorname{MAE}=\frac{1}{n} \sum_{i=1}^{n}\left|y_{i}-y_{i}^{\prime}\right|
\end{gathered}
$$

where

$n \quad=$ the number of samples in the model,

$y_{i}=$ the observed winter wheat yield,

$y_{i}^{\prime}=$ the estimated winter wheat yield.

The closer the $\mathrm{R}^{2}$ value was to 1 , the higher the model estimation accuracy; the smaller the RMSE and MAE values were, the smaller the difference between the observed yield and the predicted yield.

\section{RESULTS \\ SPATIOTEMPORAL FUSION OF GF-1 WFV AND TERRA MODIS DATA}

The ESTARFM algorithm was used for the spatiotemporal data fusion. The consistency of the input GF-1 WFV and Terra MODIS data affected the accuracy of the fusion algorithm. To verify the consistency between the input data, the surface reflectance in the red and NIR bands, NDVI, EVI2, and WDRVI of the GF-1 WFV data were aggregated to the spatial resolution of MODIS, and the consistency assessment results are shown in figure 4 . The $\mathrm{R}^{2}$ values of all indicators between the aggregated GF-1 WFV and MOD09GQ products reached 0.6. For the three vegetation

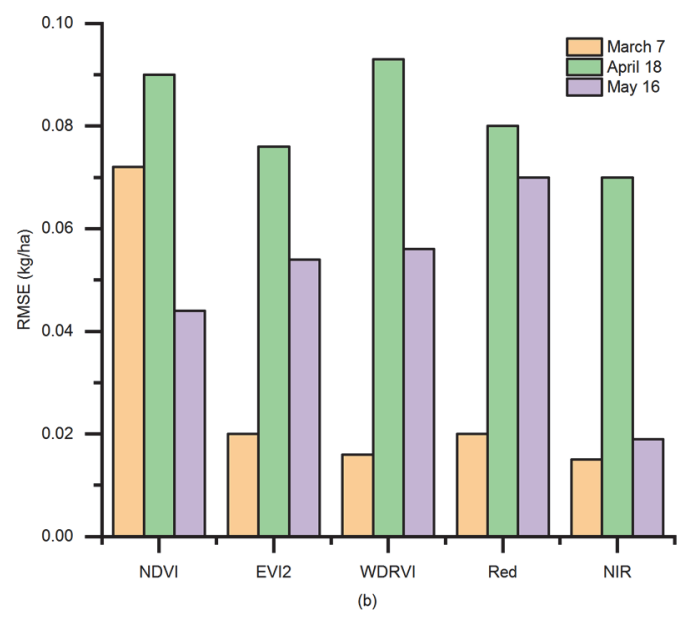

Figure 4. The $\mathbf{R}^{2}$ values and RMSEs between the GF-1 WFV and Terra MODIS data at the spatial resolution of the Terra MODIS data: (a) $\mathbf{R}^{2}$ values between indicators, and (b) RMSEs between indicators. 
indexes (NDVI, EVI2, and WDRVI), the correlations between the two data sources showed the following ranking: WDRVI > EVI2 > NDVI. The RMSEs of each indicator ranged from 0.02 to 0.09 . These results showed that the aggregated GF-1 WFV data has good consistency with the MOD09GQ product.

To verify the prediction ability of the ESTARFM algorithm for the GF-1 WFV and Terra MODIS data, the MOD09GQ product obtained on 26 April 2017 was used as the coarse-resolution image of the predicted date, and the predicted fine-resolution image was compared with the GF$1 \mathrm{WFV}$ image acquired on the same date. Furthermore, since the fine-resolution data available for verification could not cover every 8-day interval of the study period, the MOD09Q1 product was used as the coarse-resolution images of the predicted date, and the predicted fine-resolution images on 22 March, 7 April, and 1 May 2017 were aggregated to the spatial resolution of the MOD09Q1 product. Then, the consistency between the fused data and the MOD09Q1 product was tested. The consistency assessment results are shown in figure 5. On 26 April, the $R^{2}$ values of all indicators between the fused image and GF-1 WFV image ranged from 0.61 to 0.93 , and the RMSEs ranged from 0.02 to 0.09 . On the remaining three dates, the $R^{2}$ values of each indicator between the MOD09Q1 product and the fused images aggregated to the spatial resolution of MODIS ranged from 0.61 to 0.93 ; the RMSEs ranged from 0.02 to 0.09. Except on 7 April, the correlations of WDRVI between the fused data and GF-1 WFV or MODIS data were ranked as follows: WDRVI $>$ EVI2 $>$ NDVI, consistent with the ranking of the indexes between the input image pairs in ESTARFM. This shows that the fusion results of ESTARFM maintain the characteristics of the GF-1 WFV data and that the fine-spatiotemporal-resolution data fused by ESTARFM during the study period has high accuracy.

\section{COMPARISON OF THE ESTIMATION ACCURACIES OF TWO TIME Window SELECTION METHOdS}

To test the impacts of different monitoring time windows on the accuracy of the yield estimation and to identify the optimal time window, based on the four monitoring time

strategies (multiple time windows with four growth stages, multiple time windows with three growth stages, a single time window of four averaged growth stages, and a single time window of three averaged growth stages), the RFR method was used to establish winter wheat yield estimation models with six different combinations of input variables. The tenfold cross-validation method was used to verify the model accuracies, and the $\mathrm{R}^{2}$ values, RMSEs and MSEs of the models were evaluated. The results are shown in figure 6 .

When each growth stage served as a time window and all four stages were included, the six established yield estimation models with different inputs all achieved the highest $\mathrm{R}^{2}$ values (0.47-0.77) and the lowest RMSEs (592-805 kg/ha) and MSEs $(438-626 \mathrm{~kg} / \mathrm{ha})$. Thus, this is the most appropriate time strategy for estimating winter wheat yield in the study area. When only three growth stages were included in multiple time windows, the $\mathrm{R}^{2}$ values of the six models were lower, ranging from 0.23 to 0.69 . Regardless of the growth stages, when the vegetation indexes were not integrated with the meteorological data, the estimation accuracies resulting from averaging the variables from four growth stages into a single time widow $\left(R^{2}\right.$ values ranging from 0.31 to 0.41 ) were higher than those resulting from averaging the variables from three growth stages into a single time widow $\left(\mathrm{R}^{2}\right.$ values ranging from 0.24 to 0.29$)$. These results indicate that the longer the monitoring time, the higher the estimation accuracy. When the meteorological data were integrated into the vegetation indexes and the variables were averaged without distinguishing the growth stage, the estimation results were largely affected by the meteorological data, resulting in the $\mathrm{R}^{2}$ values of the single time window averaging four growth stages $(0.58-0.60)$ being slightly lower than those of the single time window averaging three growth stages (0.63-0.64).

Overall, the RMSEs ranged between 592 and $991 \mathrm{~kg} / \mathrm{ha}$, and the MSEs ranged from 438 to $807 \mathrm{~kg} / \mathrm{ha}$. These results indicate that the RFR method is suitable for winter wheat yield estimations in the study area.

Moreover, regardless of how long the monitoring time was, the models based on multiple time windows achieved higher accuracies than those based on a single time window. This means that dividing the monitoring period into multiple
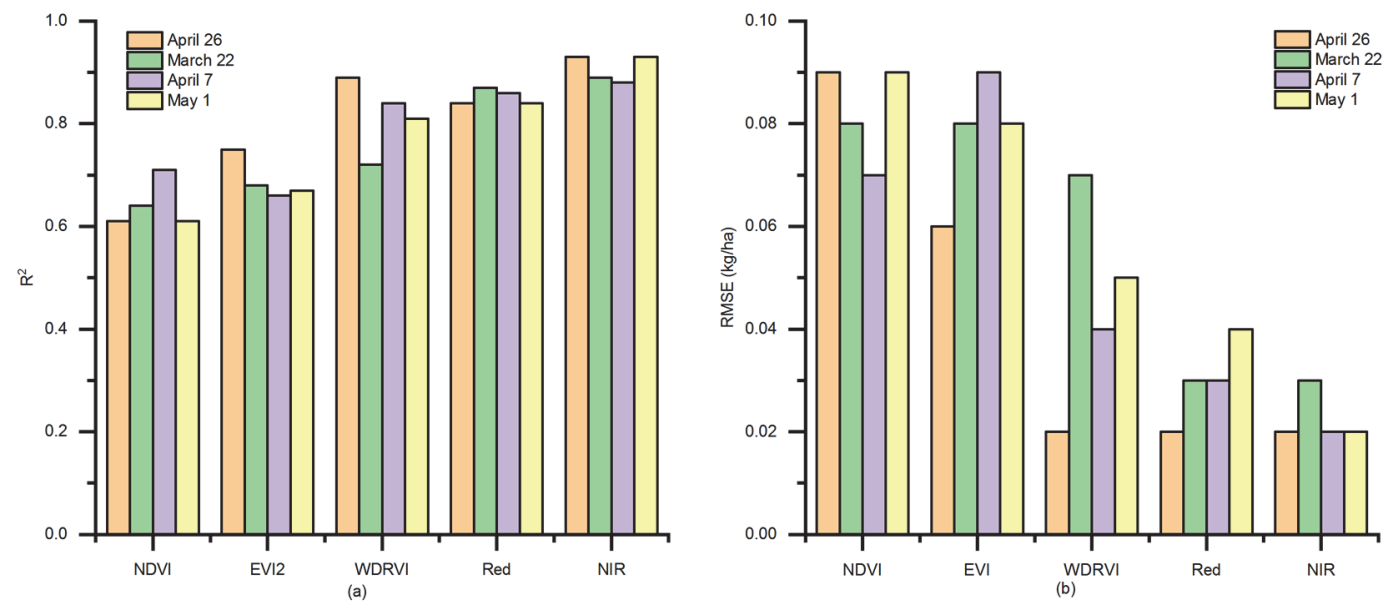

Figure 5. Accuracy assessment of the fusion results of the GF-1 WFV and Terra MODIS data by ESTARFM: (a) $\mathbf{R}^{2}$ values between indicators, and (b) RMSEs between indicators. 

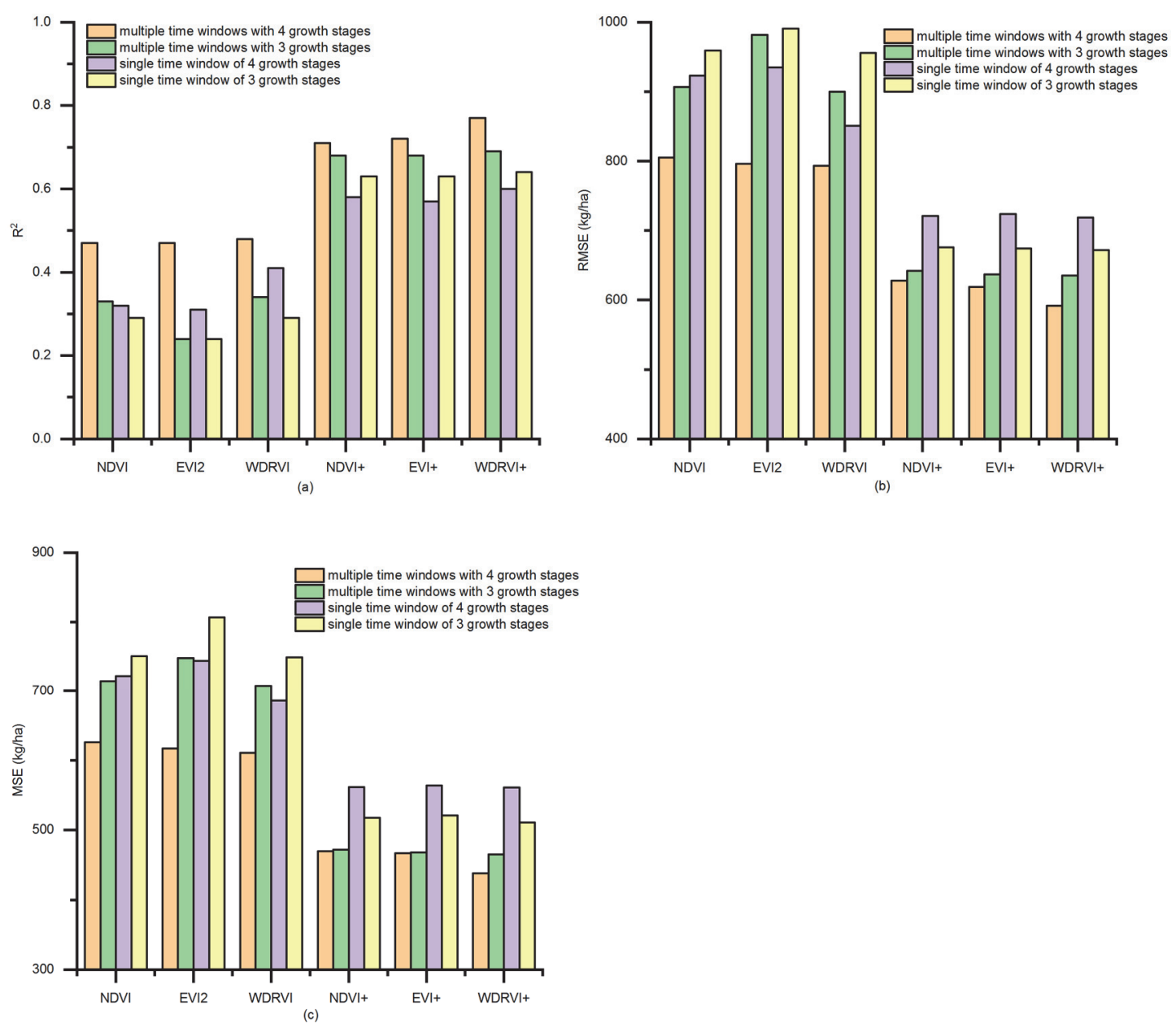

Figure 6. The accuracies of six models (NDVI+ means NDVI+ meteorology model, EVI2+ means EVI+ meteorology model, WDRVI+ means WDRVI+ meteorology model) in estimating winter wheat yield in the four time strategies based on the tenfold cross-validation results: (a) $R^{2}$ values, (b) RMSEs, (c) MSEs.

time windows according to growth stages represents the optimal time window selection method for winter wheat yield estimations in the study area.

\section{COMPARISON OF THE ESTIMATION ACCURACIES OF THREE VEGETATION INDEXES}

To test whether WDRVI can better explain the variability in winter wheat yield than other more commonly used vegetation indexes, based on the most suitable time strategy for estimating winter wheat yields determined in the above research and six different combinations of input variables, the accuracies of the RFR models are shown in table 3. The impacts of integrating meteorological factors into the models on the estimation accuracy were analyzed. Furthermore, the applicability of the three vegetation indexes of NDVI, EVI2,

Table 3. The accuracies of the RFR models with six different combinations of input variables based on the optimal time strategy.

\begin{tabular}{lccc}
\hline Model & $\mathrm{R}^{2}$ & $\begin{array}{c}\text { RMSE } \\
(\mathrm{kg} / \mathrm{ha})\end{array}$ & $\begin{array}{c}\mathrm{MSE} \\
(\mathrm{kg} / \mathrm{ha})\end{array}$ \\
\hline NDVI & 0.47 & 805 & 626 \\
EVI2 & 0.48 & 796 & 617 \\
WDRVI & 0.48 & 793 & 611 \\
NDVI+meteorology & 0.71 & 628 & 470 \\
EVI2+meteorology & 0.72 & 619 & 467 \\
WDRVI+meteorology & 0.77 & 592 & 438 \\
\hline
\end{tabular}

and WDRVI in estimating yields was evaluated. The yield estimation accuracy was significantly improved with the integration of the impacts of meteorological factors into the three vegetation indexes. The $\mathrm{R}^{2}$ values increased by more than 0.2, and the RMSEs and MSEs decreased by more than $150 \mathrm{~kg} / \mathrm{ha}$. The crop growth conditions and yield were comprehensively affected by multiple factors in complex ways, so the comprehensive consideration of remote sensing data and meteorological factors will benefit crop yield estimates in advance.

Among the three studied vegetation indexes, regardless of whether meteorological factors were integrated, the performance of WDRVI was always better than that of EVI2 and NDVI. The gap between EVI2 and NDVI was not very large. In particular, the WDRVI+ meteorology yield estimation model established by integrating the WDRVI and meteorological factors was the best model among the six winter wheat yield estimation models in the study area because its $\mathrm{R}^{2}$ value reached 0.77 , and its RMSE (592 kg/ha) and MSE $(438 \mathrm{~kg} / \mathrm{ha})$ values were the smallest. Therefore, the WDRVI is more suitable for estimations of winter wheat yield than the other two vegetation indexes in the study area. 


\section{WINTER WHEAT YiELD ESTIMATION RESULTS FROM ReMote Sensing Data WITH DiffERENT SPATIAL RESOLUTIONS}

To consider the extent to which the spatial resolutions of remote sensing images affect yield estimation results, two winter wheat yield estimation models based on fine- and coarse-spatial-resolution images were compared. The-finespatial-resolution remote sensing data were generated by the fusion of the GF-1 WFV and Terra MODIS data in this study, and the coarse-resolution remote sensing data were the 8-day synthetic product of MOD09Q1. Specifically, the growth cycle of winter wheat after overwintering in the study area was divided into four key growth stages. The WDRVI and meteorological factors at each growth stage based on the fine- and coarse-spatial-resolution images were selected as input variables, and the RFR method was used to estimate and verify the yield values. The results of the tenfold cross-validation are shown in figure 7 . Because the meteorological data used for the two spatial resolutions of the remote sensing data had the same spatial resolution of $16 \mathrm{~m}$, the gap between the yield estimation results was narrowed. However, remote sensing data with fine spatial resolutions can better estimate winter wheat yield than remote sensing data with coarse resolutions. The $\mathrm{R}^{2}$ values between the estimated yield and the observed yield were 0.77 and 0.73 , respectively. The RMSE and MSE were reduced as the spatial resolution increased. This shows that the spatial resolution of remote sensing data has a critical impact on the estimation results of winter wheat yield in the study area, and the improvement of the spatial resolution can significantly increase the estimation accuracy.

The yields estimated using the two data sources with different spatial resolutions and the observed winter wheat yield in the study area in 2017 were spatially visualized (fig. 8). The spatial pattern of winter wheat yield in the study area is uneven. The eastern part of the study area is close to the Bohai Sea, with severe soil salinization, poor agricultural production conditions, and low levels of farmland water conservancy facilities and mechanization, resulting in lower observed yields of winter wheat but higher estimates. The

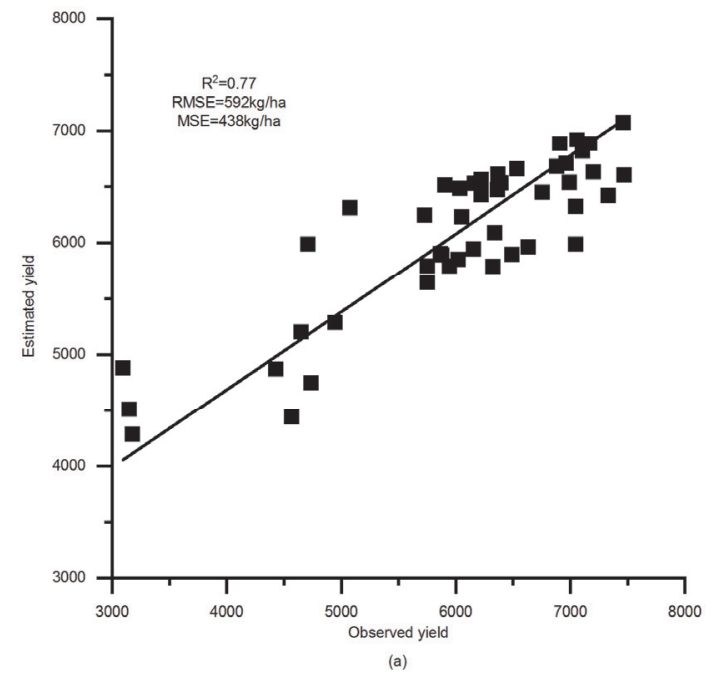

central and western parts of the study area are more suitable for farming, with flat terrain, fertile soils, suitable climates, and good irrigation conditions. Both data sources could predict the general trend of the observed yield. There were more overestimations and underestimations in the results obtained using the MODIS data. The estimation results of the finespatial-resolution data sources were closer to the observed yields than the results of the coarse-spatial-resolution data. However, some extremely high- or low-yield areas (above $7000 \mathrm{~kg} / \mathrm{ha}$, below $4000 \mathrm{~kg} / \mathrm{ha}$ ) were difficult to estimate by both remote sensing data sources.

\section{DISCUSSION}

\section{ANALYSIS OF THE ESTIMATION STRATEGIES OF WinTER WHEAT YIELD}

Both the appropriate time window selection method and the optimal vegetation index were identified in this study.

To consider whether to distinguish among growth stages during the monitoring period, the accuracy of the yield estimations obtained based on multiple time windows and those obtained based on single time windows were compared. In the models using periods of four stages or three stages, the accuracies of the multiple time windows were higher than those of the single time windows. The weight of the winter wheat growth information at each growth stage on the yield was determined by multiple weighting methods ( $\mathrm{Li}$ et al., 2014; Tian et al., 2020). Research shows that the weight of each growth stage has a great impact on the estimation accuracy of winter wheat yield. If the growth stages during the entire monitoring period are not distinguished and all monitored variables are averaged equally, the establishment of models based on single time windows will cause information loss. In contrast, the growth information will be described in more detail by recording the status and conditions at each growth stage, thereby improving the accuracies of yield estimations.

The three vegetation indexes of NDVI, EVI2 and WDRVI are all calculated using the surface reflectances of the red and NIR bands. WDRVI uses a weighting coefficient

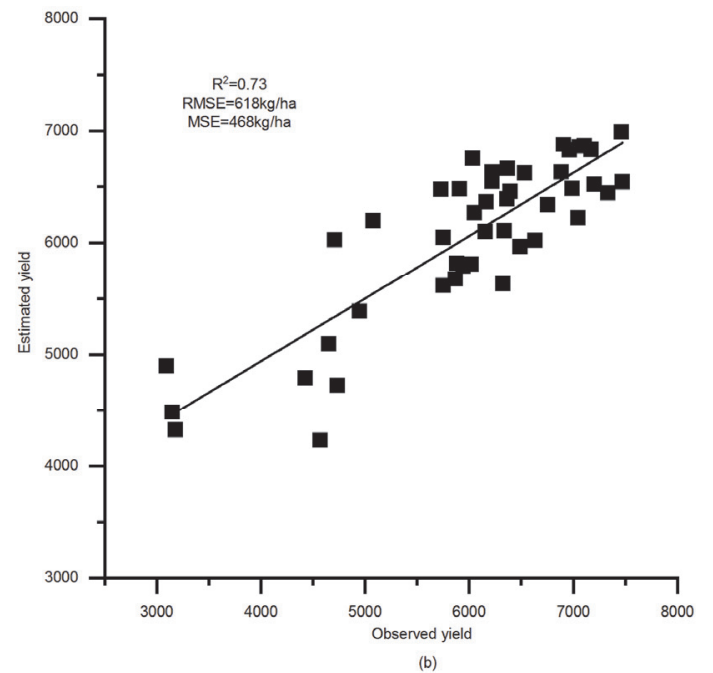

Figure 7. Estimated results of winter wheat yield from remote sensing data with two spatial resolutions: (a) fused data and (b) MOD09Q1 product. 


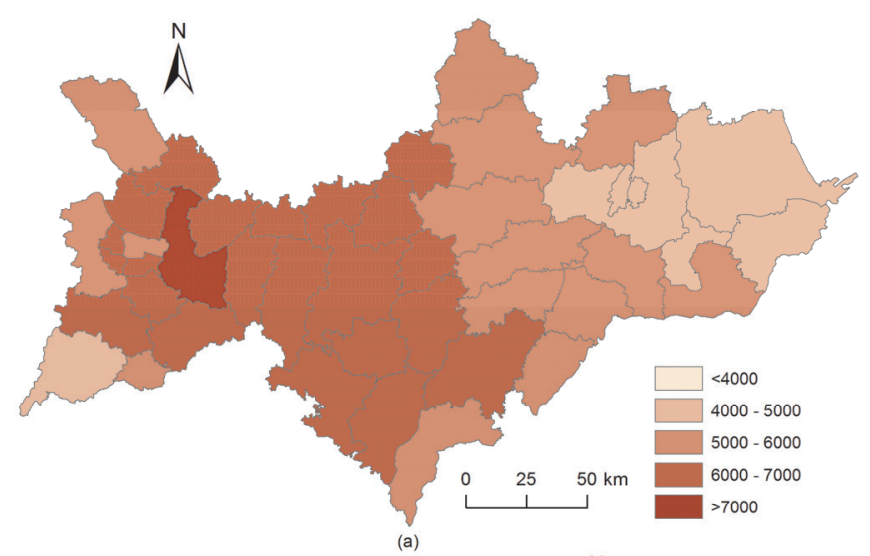

(a)

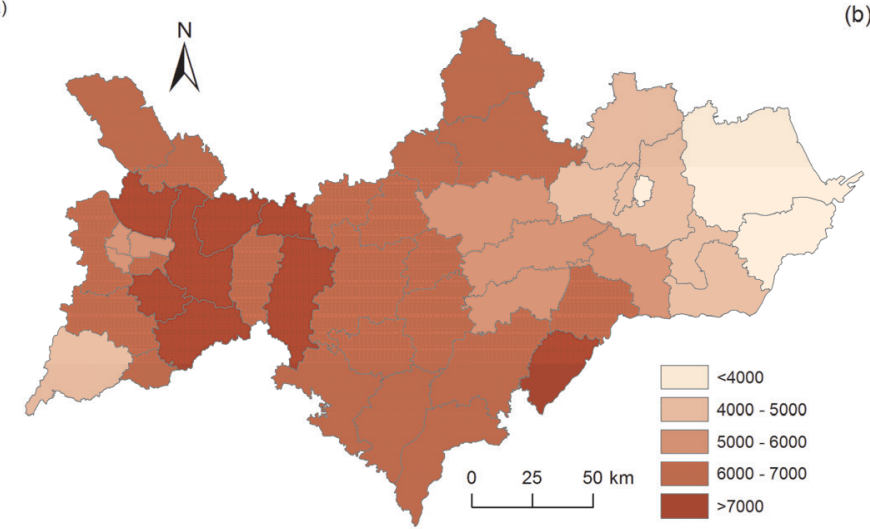

(c)

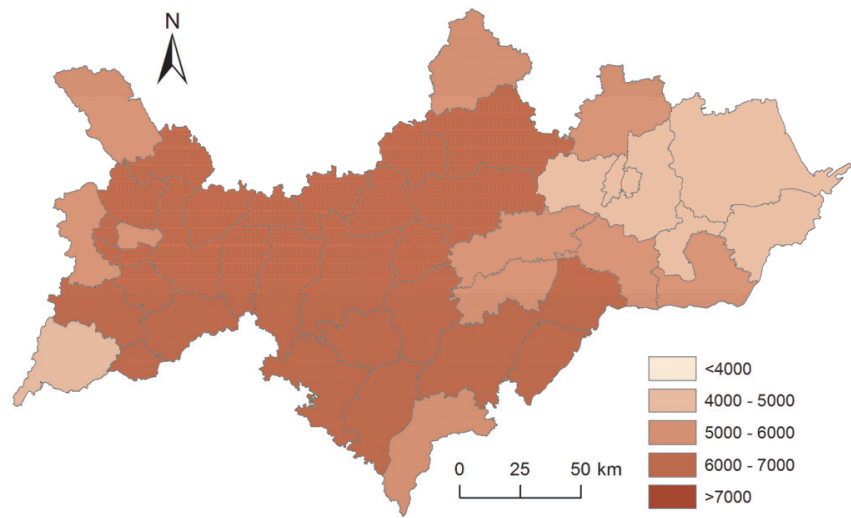

(b)

Figure 8. Spatial distributions of the observed and estimated winter wheat yields in 2017 based on the fused data of GF-1 WFV and the Terra MODIS and MOD09Q1 product: (a) fused data, (b) MOD09Q1 product, and (c) observed yield.

to narrow the gap between the contributions of the red and NIR bands to the vegetation index (Vina et al., 2004). This causes WDRVI to have the advantages of resisting atmospheric aerosols, reducing the interference of soil background values, and not being easily saturated. Bolton and Friedl (2013) found that the correlation between the vegetation index and crop yield at the county scale was the highest at 65 to 75 days after corn jointing and 80 days after soybean jointing. Sakamoto et al. (2013) found that the MODIS-WDRVI had the highest correlation with maize yield observed seven days before the silkworm period. Crops at these stages usually grow vigorously and reach medium to high LAI states, while NDVI is easily saturated at this time (Turner et al., 1999; Guo, 2017). The effects of EVI2 and WDRVI were further compared, and it was found that WDRVI is better than EVI2 in estimating winter wheat yield. Compared with NDVI and EVI2, WDRVI can more effectively reflect the spatial variability in vegetation and has a stronger ability to identify vegetation differences under medium and high LAI values. This is consistent with the research results of Dempewolf et al. (2014).

\section{THE IMPACT OF SPATIAL RESOLUTION ON YiELD ESTIMATION}

The extraction accuracy of the winter wheat planting area directly affects the accuracy of yield estimation. The arable lands in most parts of China are relatively fragmented, and the spatial resolution of MODIS data is low, resulting in winter wheat pixels extracted from MODIS data including forestland and built-up land. Serious mixed pixel problems lead to large vegetation index errors. However, the GF-1 WFV images and the fine-spatial-resolution images fused by the GF-1 WFV and Terra MODIS data contain rich detailed information on ground features, making it easier to obtain pure pixels and reduce classification errors. Therefore, the use of high-spatial-resolution images can significantly improve the estimation accuracy of winter wheat yield.

In this study, the $\mathrm{R}^{2}$ value between the estimated yield and the observed yield was increased by 0.04 , and the RMSE and MSE were decreased (fig. 7). If the meteorological data were interpolated to the same spatial resolution as the MODIS data, the gap would become larger. Li et al. (2017) estimated the LAI based on GF-1 data and showed that when the spatial resolution was increased from 16 to $30 \mathrm{~m}$, the $\mathrm{R}^{2}$ between the estimated value and the actual value decreased from 0.834 to 0.656 . The research indicated that the spatial resolution has a great impact on the LAI inversion results. Therefore, it is feasible to improve the spatial resolution of data sources through the spatiotemporal fusion of multisource remote sensing data, thereby improving the estimation accuracy of the target information.

\section{UNCERTAINTIES IN THE STUDY}

Many machine learning methods have been compared for crop yield estimations in previous studies, and the results show that the RFR method has better applicability than other methods. When multisource data, including remote sensing data and environmental data, are used for crop yield 
estimations, multiple collinearity problems may occur. The RFR model is not sensitive to multiple collinearity problems and can predict the effects of multiple variables well. Therefore, RFR is a promising method for comprehensive multifactor crop yield estimations.

In this study, the ESTARFM algorithm was used to fuse GF-1 WFV and Terra MODIS data to form sequence data with high spatial and temporal resolutions from March to May 2017. Although the consistency between the GF-1 WFV and Terra MODIS data and the accuracy of the fusion results by ESTARFM were verified before and after the spatiotemporal data fusion in this study, the spatial resolution was significantly different between the GF-1 WFV and Terra MODIS data, and the characteristic changes of land use types in coarse pixels may lead to uncertainties in the accuracy of the prediction results when coupled with the small size of cultivated land plots. For a large area of continuous farmland, it is not difficult to accurately predict images with fine spatial resolutions. However, some farmlands in the study area are mixed with woodlands and built-up areas, where inaccurate predictions may occur. With the rapid development of machine learning, many learning-based spatiotemporal data fusion algorithms have emerged (Duveiller and Cescatti, 2016; Liu et al., 2016). In the future, the accuracies of these emerging spatiotemporal data fusion algorithms will be tested to evaluate their potential for the improvement of the accuracy of yield estimations.

In addition, only remote sensing data and meteorological data were used in this study, and the lack of the inclusion of comprehensive data types is an important reason for overestimations or underestimations of winter wheat yield. For example, Haixing County, Huanghua City, and Yanshan County in the eastern part close to the Bohai Sea were overestimated due to the poor farming conditions that exist in these regions. In contrast, Zhengding County and Xinle City in the western part of the study area were underestimated. The reason may be that these counties (districts) are the core areas of the Hebei Province project area of the National Grain High-yield Science and Technology Project, where the field management practices are better than those in ordinary fields, and winter wheat yields are greatly affected by human factors. There are many factors that affect the yield of winter wheat, such as soil conditions, field management (such as winter wheat varieties, fertilizer amount, and irrigation amount), diseases and insect pests. The integration of more factors related to winter wheat yield will be the focus of future research. Furthermore, this study used the county-level winter wheat yield data recorded in statistical yearbooks to verify the yield estimation accuracies. The accuracy recorded within each county was not verified due to the lack of field survey data on winter wheat yields. In future research, the pixel-level matching of remote sensing data should be considered, and the accuracy verification needs to be improved.

\section{Conclusions}

Spatiotemporal data fusion provides an approach for obtaining time-series remote sensing data with a high spatial resolution; these time series are essential for crop growth monitoring and yield estimations. In this article, the potential of the spatiotemporal fusion of GF-1 WFV and Terra MODIS data was demonstrated. On this basis, a suitable time window selection method and an optimal vegetation index for winter wheat yield estimation were provided. The results of this article show the following conclusions.

- The ESTARFM algorithm is suitable for the fusion of GF-1 WFV and Terra MODIS data in the study area, and the fusion results can maintain the characteristics of GF-1 WFV data.

- The RFR method can be applied to winter wheat yield estimations in the study area well. The application of a longer monitoring time can provide a higher accuracy for the model. The results of dividing the growth cycle into multiple time windows according to the growth stages in yield estimations are better than those obtained when taking the whole monitoring period as a single time window, and the adoption of multiple time windows representing the growth information at the four growth stages is the most suitable time strategy for winter wheat yield estimations in the study area.

- The estimation ability of WDRVI is better than those of EVI2 and NDVI. The WDRVI+ meteorological RFR model $\left(\mathrm{R}^{2}=0.77, \mathrm{RSME}=592 \mathrm{~kg} / \mathrm{ha}, \mathrm{MSE}=\right.$ $438 \mathrm{~kg} / \mathrm{ha}$ ) established based on four growth stages is the most suitable model.

- The regional distribution of winter wheat yield in the study area is uneven, with the western and central regions having significantly higher winter wheat yields than the eastern regions. High-spatial-resolution images can significantly improve the estimation accuracy of winter wheat yield, especially for extremely high-yield areas.

\section{REFERENCES}

Alberto, G. S., Juan, F. S., \& Waldo, O. B. (2014). Predictive ability of machine learning methods for massive crop yield prediction. Span. J. Agric. Res. , 12, 313-328. https://doi.org/10.5424/sjar/2014122-4439

Beniaich, A., Naves Silva, M. L., Avalos, F. A., Menezes, M. D., \& Candido, B. M. (2019). Determination of vegetation cover index under different soil management systems of cover plants by using an unmanned aerial vehicle with an onboard digital photographic camera. Semina-Ciencias Agrarias, 40(1), 49-66. https://doi.org/10.5433/1679-0359.2019v40n1p49

Bolton, D. K., \& Friedl, M. A. (2013). Forecasting crop yield using remotely sensed vegetation indices and crop phenology metrics. Agric. For. Meteorol., 173, 74-84. https://doi.org/10.1016/j.agrformet.2013.01.007

Breiman, L. (2001). Random forests. Machine Learning, 45(1), 532. https://doi.org/10.1023/A:1010933404324

Chen, Y., McVicar, T. R., Donohue, R. J., Garg, N., Waldner, F., Ota, N.,... Lawes, R. (2020). To blend or not to blend? A framework for nationwide landsat-MODIS data selection for crop yield prediction. Remote Sensing, 12(10), 1653. https://doi.org/10.3390/rs12101653 
Chen, Z., Ren, J., Tang, H., Shi, Y., Leng, P., Liu, J.,... Hasiyuya, Y. (2016). Progress and perspectives on agricultural remote sensing research and applications in China. J. Remote Sens., 20(5), 748767.

Curtis, T., \& Halford, N. G. (2014). Food security: The challenge of increasing wheat yield and the importance of not compromising food safety. Ann. Appl. Biol., 164(3), 354-372. https://doi.org/10.1111/aab.12108

Dempewolf, J., Adusei, B., Becker-Reshef, I., Hansen, M., Potapov, P., Khan, A., \& Barker, B. (2014). Wheat yield forecasting for Punjab Province from vegetation index time series and historic crop statistics. Remote Sensing, 6(10), 9653-9675. https://doi.org/10.3390/rs6109653

Du, H. J., Liu, Q., Li, J., \& Yang, L. (2013). Retrieving crop leaf area index by combining optical and microwave vegetation indices: A feasibility analysis. J. Appl. Remote Sens., 17(6), 1587-1611.

Duveiller, G., \& Cescatti, A. (2016). Spatially downscaling suninduced chlorophyll fluorescence leads to an improved temporal correlation with gross primary productivity. Remote Sens. Environ., 182, 72-89. https://doi.org/10.1016/j.rse.2016.04.027

Emelyanova, I. V., McVicar, T. R., Van Niel, T. G., Li, L. T., \& van Dijk, A. I. (2013). Assessing the accuracy of blending Landsat-MODIS surface reflectances in two landscapes with contrasting spatial and temporal dynamics: A framework for algorithm selection. Remote Sens. Environ., 133, 193-209. https://doi.org/10.1016/j.rse.2013.02.007

Franz, T. E., Pokal, S., Gibson, J. P., Zhou, Y., Gholizadeh, H., Tenorio, F. A.,... Wardlow, B. (2020). The role of topography, soil, and remotely sensed vegetation condition towards predicting crop yield. Field Crops Res., 252, 107788. https://doi.org/10.1016/j.fcr.2020.107788

Gao, F., \& Anderson, M. (2019). Evaluating yield variability of corn and soybean using Landsat-8, Sentinel-2 and Modis in Google Earth Engine. Proc. IEEE Int. Geoscience and Remote Sensing Symp. (pp. 7286-7289). IEEE. https://doi.org/10.1109/IGARSS.2019.8897990

Gao, F., Anderson, M., Daughtry, C., \& Johnson, D. (2018). Assessing the variability of corn and soybean yields in central Iowa using high spatiotemporal resolution multi-satellite imagery. Remote Sensing, 10(9), 1489. https://doi.org/10.3390/rs10091489

Gao, F., Masek, J., Schwaller, M., \& Hall, F. (2006). On the blending of the Landsat and MODIS surface reflectance: Predicting daily Landsat surface reflectance. IEEE Trans. Geosci. Remote Sens., 44(8), 2207-2218. https://doi.org/10.1109/TGRS.2006.872081

Gitelson, A. A. (2004). Wide dynamic range vegetation index for remote quantification of biophysical characteristics of vegetation. J. Plant Physiol., 161(2), 165-173. https://doi.org/10.1078/0176-1617-01176

Guo, P. (2017). Research on key parameter extraction method of farm crops growing based on low altitude airborne sensor technology. PhD diss. Beijing: China University of Geosciences, School of Earth Science and Resources.

Han, J., Zhang, Z., Cao, J., Luo, Y., Zhang, L., Li, Z., \& Zhang, J. (2020). Prediction of winter wheat yield based on multi-source data and machine learning in China. Remote Sensing, 12(2), 236. https://doi.org/10.3390/rs12020236

He, Z., Xia, X., \& Zhang, Y. (2010). Breeding noodle wheat in China. In Asian noodles: Science, technology, and processing (pp. 1-23). Hoboken, NJ: Wiley online. https://doi.org/10.1002/9780470634370.ch1

Huang, J., Luo, Q., Liu, X., \& Zhang, J. (2016). Winter wheat yield forecasting based on time series of MODIS NDVI. Trans. CSAM, 47(2), 295-301.
Jiang, Z., Chen, J., Chen, J., Liu, J., Ren, J., Li, H.,... Li, H. (2014). Application of crop model data assimilation with a particle filter for estimating regional winter wheat yields. IEEE J. Selected Topics Appl., 7(11), 4422-4431. https://doi.org/10.1109/JSTARS.2014.2316012

Jiang, Z., Huete, A. R., Didan, K., \& Miura, T. (2008). Development of a two-band enhanced vegetation index without a blue band. Remote Sens. Environ., 112(10), 3833-3845. https://doi.org/10.1016/j.rse.2008.06.006

Karthikeyan, L., Chawla, I., \& Mishra, A. K. (2020). A review of remote sensing applications in agriculture for food security: Crop growth and yield, irrigation, and crop losses. J. Hydrol., 586, 124905. https://doi.org/10.1016/j.jhydrol.2020.124905

Kern, A., Barcza, Z., Marjanovic, H., Arendas, T., Fodor, N., Bonis, P.,... Lichtenberger, J. (2018). Statistical modelling of crop yield in Central Europe using climate data and remote sensing vegetation indices. Agric. For. Meteorol., 260-261, 300-320. https://doi.org/10.1016/j.agrformet.2018.06.009

Khanizadeh, S., Rekika, D., Levasseur, A., Groleau, Y., Richer, C., $\&$ Fisher, H. (2005). The effects of different cultural and environmental factors on grapevine growth, winter hardiness and performance, in three locations, in Canada. Small Fruits Rev., 4(3), 3-28. https://doi.org/10.1300/J301v04n03_02

Kong, F., Li, X., Wang, H., Xie, D., Li, X., \& Bai, Y. (2016). Land cover classification based on fused data from GF-1 and MODIS NDVI time series. Remote Sensing, 8(9), 741. https://doi.org/10.3390/rs8090741

Li, H., Chen, Z.-X., Jiang, Z.-w., Wu, W.-b., Ren, J.-q., Liu, B., \& Tuya, H. (2017). Comparative analysis of GF-1, HJ-1, and Landsat- 8 data for estimating the leaf area index of winter wheat. J. Integrative Agric., 16(2), 266-285. https://doi.org/10.1016/S2095-3119(15)61293-X

Li, Y., Wang, P., Liu, J., Zhang, S., \& Li, L. (2014). Evaluation of drought monitoring effects in the main growing stages of winter wheat by using the vegetation temperature condition index-II. Agric. Res. Arid Areas, 32(1), 236-239.

Lin, Y., \& Shao, H. (2020). Study on optimal time and influencing factors for winter wheat yield prediction in Henan based on random forest algorithm. J. Triticeae Crops, 40(7), 874-880.

Liu, X., Deng, C., Wang, S., Huang, G., Zhao, B., \& Lauren, P. (2016). Fast and accurate spatiotemporal fusion based upon extreme learning machine. IEEE Geosci. Remote. S., 13(12), 2039-2043. https://doi.org/10.1109/LGRS.2016.2622726

Lopez, M. A., Lombardo, J. M., Lopez, M., Alvarez, D., Velasco, S., \& Terron, S. (2020). Traceable ecosystem and strategic framework for the creation of an integrated pest management system for intensive farming. Int. J. Interactive Multimedia Artificial Intelligence, 6(3), 47-54. https://doi.org/10.9781/ijimai.2020.08.004

Pan, H., Chen, Z., de Wit, A., \& Ren, J. (2019). Joint assimilation of leaf area index and soil moisture from Sentinel-1 and Sentinel-2 Data into the WOFOST model for winter wheat yield estimation. Sensors, 19(14), 3161. https://doi.org/10.3390/s19143161

Rastgou, M., Bayat, H., Mansoorizadeh, M., \& Gregory, A. S. (2020). Estimating the soil water retention curve: Comparison of multiple nonlinear regression approach and random forest data mining technique. Comput. Electron. Agric., 174, 105502. https://doi.org/10.1016/j.compag.2020.105502

Sakamoto, T. (2020). Incorporating environmental variables into a MODIS-based crop yield estimation method for United States corn and soybeans through the use of a random forest regression algorithm. ISPRS J. Photogramm. Remote Sens., 160, 208-228. https://doi.org/10.1016/j.isprsjprs.2019.12.012 
Sakamoto, T., Gitelson, A. A., \& Arkebauer, T. J. (2013). MODISbased corn grain yield estimation model incorporating crop phenology information. Remote Sens. Environ., 131, 215-231. https://doi.org/10.1016/j.rse.2012.12.017

Tao, G., Jia, K., Zhao, X., Wei, X., Xie, X., Zhang, X.,... Zhang, X. (2019). Generating high spatio-temporal resolution fractional vegetation cover by fusing GF-1 WFV and MODIS Data. Remote Sensing, 11(19), 2324. https://doi.org/10.3390/rs11192324

Tian, H., Wang, P., Tansey, K., Zhang, S., Zhang, J., \& Li, H. (2020). An IPSO-BP neural network for estimating wheat yield using two remotely sensed variables in the Guanzhong Plain, PR China. Comput. Electron. Agric., 169, 105180. https://doi.org/10.1016/j.compag.2019.105180

Turner, D. P., Cohen, W. B., Kennedy, R. E., Fassnacht, K. S., \& Briggs, J. M. (1999). Relationships between leaf area index and Landsat TM spectral vegetation indices across three temperate zone sites. Remote Sens. Environ., 70(1), 52-68. https://doi.org/10.1016/S0034-4257(99)00057-7

Vina, A., Henebry, G. M., \& Gitelson, A. A. (2004). Satellite monitoring of vegetation dynamics: Sensitivity enhancement by the wide dynamic range vegetation index. Geophys. Res. Lett., 31(4). https://doi.org/10.1029/2003GL019034

Wang, L., Wang, P., Liang, S., Zhu, Y., Khan, J., \& Fang, S. (2020). Monitoring maize growth on the North China Plain using a hybrid genetic algorithm-based back-propagation neural network model. Comput. Electron. Agric., 170, 105238. https://doi.org/10.1016/j.compag.2020.105238

Wang, M., Tao, F.-1., \& Shi, W.-j. (2014). Corn yield forecasting in northeast china using remotely sensed spectral indices and crop phenology metrics. J. Integrative Agric., 13(7), 1538-1545. https://oi.org/10.1016/S2095-3119(14)60817-0

Wheeler, T. R., Craufurd, P. Q., Ellis, R. H., Porter, J. R., \& Vara Prasad, P. V. (2000). Temperature variability and the yield of annual crops. Agric. Ecosyst. Environ., 82(1), 159-167. https://doi.org/10.1016/S0167-8809(00)00224-3
Xu, C., Qu, J. J., Hao, X., Cosh, M. H., Zhu, Z., \& Gutenberg, L. (2020). Monitoring crop water content for corn and soybean fields through data fusion of MODIS and Landsat measurements in Iowa. Agric. Water Manag., 227, 105844. https://doi.org/10.1016/j.agwat.2019.105844

Zhang, L., Guo, C. L., Zhao, L. Y., Zhu, Y., Cao, W. X., Tian, Y. C.,... Wang, X. (2016). Estimating wheat yield by integrating the WheatGrow and PROSAIL models. Field Crops Res., 192, 5566. https://doi.org/10.1016/j.fcr.2016.04.014

Zhang, S., Zhao, G., Lang, K., Su, B., Chen, X., Xi, X., \& Zhang, H. (2019). Integrated satellite, unmanned aerial vehicle (UAV) and ground inversion of the SPAD of winter wheat in the reviving stage. Sensors, 19(7), 1485. https://doi.org/10.3390/s19071485

Zhao, G., Zhang, Y., Tan, J., Li, C., \& Ren, Y. (2020). A data fusion modeling framework for retrieval of land surface temperature from Landsat- 8 and MODIS Data. Sensors, 20(15), 4337. https://doi.org/10.3390/s20154337

Zhou, X., Wang, P., Tansey, K., Zhang, S., Li, H., \& Wang, L. (2020). Developing a fused vegetation temperature condition index for drought monitoring at field scales using Sentinel-2 and MODIS imagery. Comput. Electron. Agric., 168, 105144. https://doi.org/10.1016/j.compag.2019.105144

Zhu, X., Chen, J., Gao, F., Chen, X., \& Masek, J. G. (2010). An enhanced spatial and temporal adaptive reflectance fusion model for complex heterogeneous regions. Remote Sens. Environ., 114(11), 2610-2623. https://doi.org/10.1016/j.rse.2010.05.032

Zou, X., \& Mottus, M. (2017). Sensitivity of common vegetation indices to the canopy structure of field crops. Remote Sensing, 9(10), 994. https://doi.org/10.3390/rs9100994

Zouggar, S. T., \& Adla, A. (2019). A diversity-accuracy measure for homogenous ensemble selection. Int. J. Interact. Multi., 5(5), 63-70. https://doi.org/10.9781/ijimai.2018.06.005 